\title{
Efecto del magnesio en el rendimiento y contenido de gluten en trigo (Triticum aestivum $L$.) en un suelo andisol
}

\author{
Effect of magnesium on the yield and gluten content \\ of wheat (Triticum aestivum L.) in an andisol soil \\ Hernán Pinilla Quezada ${ }^{1}$, Luis Herrera Floody ${ }^{2}$, Rodrigo Benavente I. ${ }^{3}$, Héctor Sanhueza Roa ${ }^{4}$
}

\section{RESUMEN}

Se evaluó el efecto de las aplicaciones de magnesio en el rendimiento, contenido de gluten del trigo y su disponibilidad en un suelo volcánico de la IX Región durante tres temporadas. No se produjeron diferencias significativas de rendimiento por efecto de las distintas dosis de magnesio en ninguno de los sitios evaluados. No se mostraron diferencias en el contenido de gluten en los años 2005 y 2007, mientras que en 2006 se registró un incremento moderado respecto al testigo. Al aplicar $1 \mathrm{~kg} \mathrm{MgO} / \mathrm{ha}$ en el suelo, se produjo un aumento promedio de $0,0032 \mathrm{cmol} / \mathrm{kg}$ de $\mathrm{Mg}$ a $0-20 \mathrm{~cm}$.

Palabras clave: Triticum aestivum, trigo, magnesio, rendimiento, gluten.

\begin{abstract}
The effect of the applications of magnesium on yield, content of gluten in wheat and its availability in a volcanic soil of the region $I X$, for three seasons was evaluated. There were no significant differences in yield, by effect of different doses of magnesium in any of the sites evaluated. Showed no differences in the gluten content in years 2005 and 2007, whereas in 2006 there was a moderate increase, respect the cero dose. In applying $1 \mathrm{~kg} \mathrm{MgO/ha}$ in soil, there was an average increase of 0,0032 $\mathrm{cmol} / \mathrm{kg}$ the $M g$ at $0-20 \mathrm{~cm}$.
\end{abstract}

Key words: Triticum aestivum, wheat, magnesium, yield, gluten.

\section{Introducción}

Los cereales son considerados, en comparación a los frutales, como cultivos de baja necesidad de magnesio. Esto, debido a que sus requerimientos varían entre 15 a $30 \mathrm{~kg}$ de $\mathrm{Mg} / \mathrm{ha}$ y por la baja incidencia de este nutriente en el rendimiento (Hart, 2000). No obstante, el Mg puede llegar a constituirse en un factor limitante, situación que se ha manifestado en algunas regiones de Europa en los últimos años (Ross, 2006).

El magnesio es parte de la molécula de clorofila, siendo esencial en el proceso de fotosíntesis para la producción de carbohidratos (Tisdale y Nelson, 1993), teniendo gran influencia en el peso del grano y responsable del color verde de la planta. Dos factores que influencian la nutrición con magnesio son el nivel de potasio y la temperatura del suelo (Singh y Pathak, 2003). Estos últimos autores demostraron que a medida que la dosis de potasio aumenta, la absorción de magnesio disminuye, generando un efecto antagónico de absorción K-Mg. De este modo, en suelos con bajo contenido de magnesio se puede inducir una deficiencia de este nutriente por aplicaciones de potasio. Por otro lado, las bajas temperaturas del suelo en la zona radicular han mostrado una disminución en la absorción de magnesio (Ross, 2006).

En Chile existe una amplia información referente al manejo de los suelos ácidos y al uso de nutrientes como el nitrógeno, fósforo, potasio, azufre y boro en la producción de cereales. Sin embargo, no se ha investigado mayormente el grado de respuesta que presenta el magnesio tanto en rendimiento como en

\footnotetext{
Ingeniero Agrónomo, Magíster en Fertilidad de Suelos, Académico Investigador FCAF. Universidad de La Frontera. E-mail: hpin@ufro.cl

2 Ingeniero Agrónomo, Magíster en Gestión y Manejo Productivo de Cereales. E-mail: herreral@ufro.cl

3 Ingeniero Agrónomo, FCAF. Universidad de La Frontera, E-mail: rbenave@ufro.cl

4 Ingeniero Ejecución Agrícola, Académico Investigador FCAF. Universidad de La Frontera. E-mail: hsanhue@ufro.cl
} 
la calidad del grano de trigo, ni respecto del nivel crítico de magnesio del suelo para el trigo. De acuerdo a lo señalado, el propósito de la presente investigación fue generar información que permita dar una respuesta racional al uso del magnesio en trigo, en suelos con niveles de magnesio intercambiable entre 0,35 a $0,45 \mathrm{cmol} / \mathrm{kg}$.

\section{Materiales y Métodos}

El experimento se realizó durante tres temporadas, 2005, 2006 y 2007, en un suelo andisol de la serie Freire, IX Región, descrito por Mella y Künhe (1985), en el área agroclimática del llano central descrita por Rouanet (1989) como mediterráneo frío. Sus principales características químicas se indican en la Tabla 1.

Tabla 1. Características químicas del suelo utilizado.

\begin{tabular}{lccc}
\hline Elemento & 2005 & 2006 & 2007 \\
\hline pH en agua & 5,78 & 5,78 & 5,78 \\
M. Orgánica (\%) & 15 & 19 & 15 \\
P Olsen $(\mathrm{mg} / \mathrm{kg})$ & 11 & 7 & 12 \\
$\mathrm{~K}(\mathrm{mg} / \mathrm{kg})$ & 158 & 219 & 158 \\
$\mathrm{Na}(\mathrm{cmol} / \mathrm{kg})$ & 0,25 & 0,28 & 0,25 \\
$\mathrm{Ca}(\mathrm{cmol} / \mathrm{kg})$ & 2,77 & 2,77 & 2,77 \\
$\mathrm{Mg}(\mathrm{cmol} / \mathrm{kg})$ & 0,35 & 0,35 & 0,45 \\
$\mathrm{Al}(\mathrm{cmol} / \mathrm{kg})$ & 0,11 & 0,14 & 0,11 \\
$\mathrm{Saturación} \mathrm{de} \mathrm{Al}(\%)$ & 2,8 & 3,28 & 2,8 \\
$\mathrm{CICE}(\mathrm{cmol} / \mathrm{kg})$ & 3,91 & 4,27 & 3,91 \\
$\mathrm{~S} . \mathrm{Bases}(\mathrm{cmol} / \mathrm{kg})$ & 3,77 & 4,13 & 3,87 \\
$\mathrm{~B}(\mathrm{mg} / \mathrm{kg})$ & 0,56 & 0,54 & 0,56 \\
Zn $(\mathrm{mg} / \mathrm{kg})$ & 0,63 & 0,63 & 0,63 \\
$\mathrm{~S}(\mathrm{mg} / \mathrm{kg})$ & 11 & 11 & 11 \\
\hline
\end{tabular}

El ensayo constó de cuatro tratamientos incluido el testigo y cuatro repeticiones (Tabla 2).

Tabla 2. Tratamientos evaluados en los ensayos de campo.

\begin{tabular}{l} 
Tratamientos \\
\hline 1. Fertilización Base $+0 \mathrm{~kg} \mathrm{MgO} / \mathrm{ha}$ \\
2. Fertilización Base $+15 \mathrm{~kg} \mathrm{MgO} / \mathrm{ha}$ \\
3. Fertilización Base $+30 \mathrm{~kg} \mathrm{MgO} / \mathrm{ha}$ \\
4. Fertilización Base $+60 \mathrm{~kg} \mathrm{MgO} / \mathrm{ha}$ \\
\hline
\end{tabular}

El diseño experimental fue en bloques distribuidos completamente al azar, en parcelas de $15 \mathrm{~m}^{2}$ $(2,5$ x 6). Se utilizó la variedad Dollinco INIA de hábito de desarrollo alternativo, sembrados entre el 7 y 10 de julio. El trigo se fertilizó a la siembra con $28 \mathrm{~kg} \mathrm{~N} / \mathrm{ha} ; 200 \mathrm{~kg} \mathrm{P}_{2} \mathrm{O}_{5} / \mathrm{ha} ; 75 \mathrm{~kg} \mathrm{~K} / 2 \mathrm{O} / \mathrm{ha}$ y $2 \mathrm{~kg} \mathrm{~B} / \mathrm{ha}$. La mezcla de fertilizante se realizó con $160 \mathrm{~kg}$ de fosfato monoamónico, $255 \mathrm{~kg}$ de superfosfato triple, $80 \mathrm{~kg}$ de nitrato de potasio, $65 \mathrm{~kg}$ de cloruro de potasio y $20 \mathrm{~kg}$ de boronat 32 . El magnesio se aplicó con magnesul en la mezcla de siembra, según cada tratamiento. Con el objeto de evitar el efecto del azufre que está presente en el sulfato de magnesio, se aplicó fertiyeso en polvo hasta alcanzar una dosis pareja de $48 \mathrm{~kg}$ de S/ha en todas las parcelas experimentales.

Del total de la dosis de $\mathrm{N}$ aplicado a la siembra un 50\% fue nitrógeno nítrico y el resto amoniacal. El resto del N hasta completar 203 kilos se realizó con 70 kilos de $\mathrm{N}$ con urea al estado de Zadoks 21 y 105 kilos de $\mathrm{N}$ con supernitro 25 a Zadoks 30.

Las variables medidas fueron rendimiento de grano, contenido de gluten húmedo y disponibilidad de magnesio en la estrata $0-20 \mathrm{~cm}$, a la cosecha. Previo al muestreo del suelo se homogeneizaron las parcelas con un motocultivador, en atención a que el magnesio se aplicó en el surco de siembra.

Luego de verificar homogeneidad de varianza y distribución normal de los datos, se efectuó un análisis de varianza utilizando SPSS (2000). Las medias se compararon con la prueba de Tuckey $(\mathrm{p} \leq 0,05)$.

\section{Resultados y Discusión}

\section{Rendimiento del grano}

De acuerdo al análisis de varianza se concluye que no existen diferencias significativas de rendimiento por efecto de los diferentes tratamientos evaluados (Figura 1).

No se observó un incremento de rendimiento por efecto de las dosis crecientes de magnesio aplicadas en los tres años evaluados. De acuerdo al análisis químico, los niveles de $\mathrm{Mg}$ en el suelo fueron de $0,35,0,35$ y $0,45 \mathrm{cmol} / \mathrm{kg}$ para los años 2005, 2006 y 2007, respectivamente; de esta forma, los resultados se contradicen con el estándar publicado por Rodríguez (1993), quien señala un nivel de suficiencia de $0,8 \mathrm{cmol} / \mathrm{kg}$ de magnesio en el suelo.

Por otra parte, los resultados se contraponen a lo expuesto por Hart (2000), quien señala que contenidos inferiores a $0,5 \mathrm{cmol} / \mathrm{kg}$ de este nutriente son deficitarios para la producción de trigo.

Con los resultados obtenidos se podría inferir que el nivel crítico de respuesta a $\mathrm{Mg}$ en trigo 


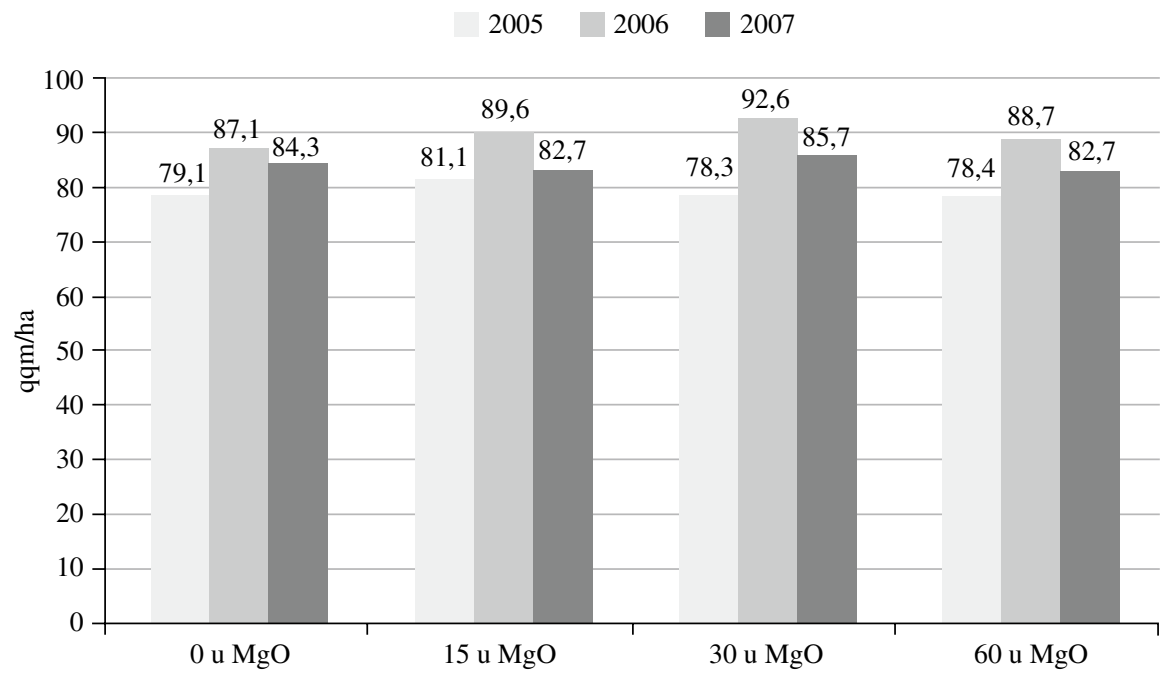

Figura 1. Efecto de las distintas dosis de magnesio evaluadas en el rendimiento del grano en temporadas 2005,2006 y 2007.

debería estar bajo los $0,35 \mathrm{cmol} / \mathrm{kg}$, lo cual concuerda con lo propuesto por Rodríguez et al. (2001) quienes señalan como nivel crítico $0,25 \mathrm{cmol} / \mathrm{kg}$ de magnesio.

Referente a la dosis de este nutriente, Hart (2000) recomienda dosis de $26 \mathrm{~kg}$ de $\mathrm{MgO} /$ ha para trigos en suelos deficientes y en Argentina, Melgar (2005) obtuvo aumentos en rendimiento aplicando $33 \mathrm{~kg}$ de $\mathrm{MgO} / \mathrm{ha}$.

\section{Gluten húmedo}

Los resultados indican que en el año 2005 se produjo un incremento en el contenido de gluten al aplicar magnesio con respecto al testigo, sin embargo, las dosis crecientes no produjeron un incremento sostenido de esta variable. En las otras dos temporadas la adición de magnesio no afectó el contenido de gluten húmedo (Figura 2).

En el caso de los años 2006 y 2007 los resultados coinciden con lo señalado por Zhao (1999), DuPont y Altenbach (2003) y Wieser (2004), quienes han indicado que el contenido de gluten del grano de trigo es determinado por la genética, ambiente y por la nutrición con nitrógeno, ya que este elemento es componente fundamental de todos los aminoácidos de las proteínas que componen el gluten. Otros elementos, como el magnesio, no tendrían influencia

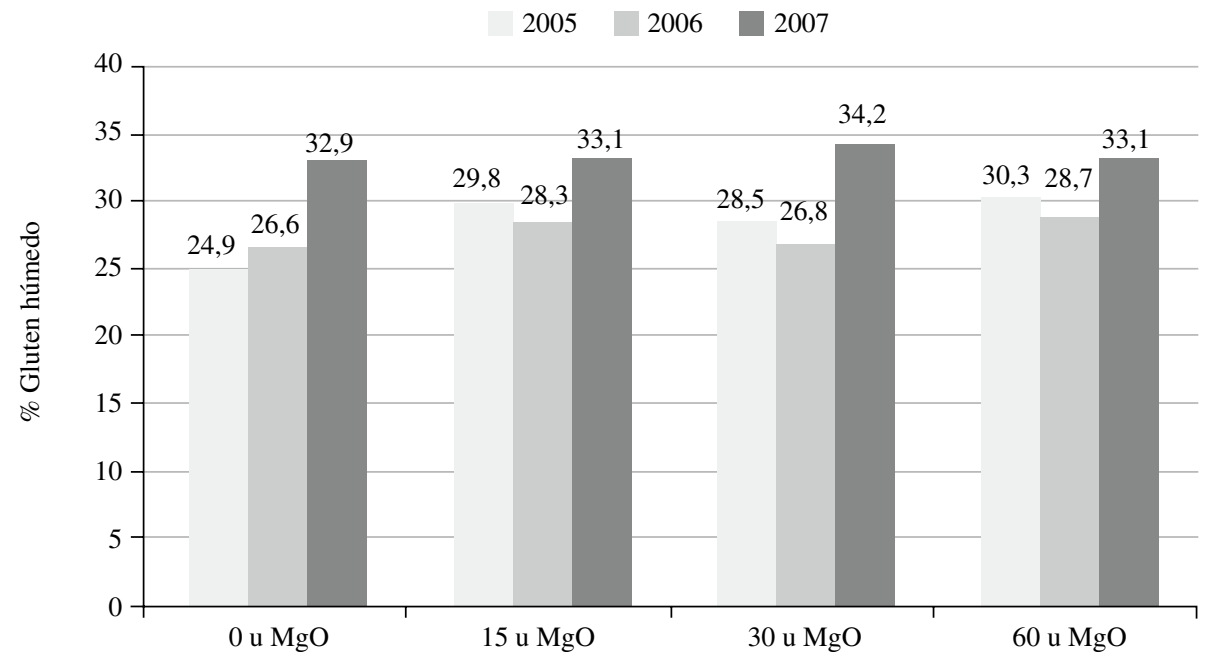

Figura 2. Efecto de las distintas dosis de magnesio en contenido de gluten húmedo en las temporadas 2005,2006 y 2007. 
en el contenido de gluten, ya que no forma parte de los aminoácidos ni participa en su formación directamente. En este sentido, es necesario hacer presente que la nutrición con $\mathrm{S}$ del trigo tiene un claro efecto en la composición de las proteínas del gluten, pero de poco o nulo efecto en su contenido total en el grano, ya que el azufre sólo es componente de los aminoácidos azufrados metionina y cisteína (Flaete, 2005, Tea et al., 2005).

En el caso del año 2005, el incremento en el contenido de gluten con respecto al testigo se contrapone con lo señalado; sin embargo, las dosis crecientes de magnesio no mostraron un incremento significativo, lo que concuerda con la literatura.

Es interesante destacar la experiencia de Singh y Pathak (2003), quienes afirman que al aplicar una fertilización equilibrada entre 62 a $70 \mathrm{~kg} \mathrm{~K} 2 \mathrm{O} /$ ha y 32 a $34 \mathrm{~kg} \mathrm{MgO} / \mathrm{ha}$, se conseguirían incrementos en el rendimiento de grano, en el contenido de almidón y proteína total del grano, sin embargo, una disminución del aminoácido lisina. De esta forma, este incremento podría deberse a un efecto de la combinación producida entre la fertilización potásica base a la siembra y la adición de magnesio de los tratamientos.

\section{Disponibilidad de magnesio en el suelo}

En la Figura 3 se presentan los resultados de la disponibilidad de magnesio en el suelo a la co- secha, por efecto de las diferentes dosis y suelos utilizados.

Los resultados en los años 2005 y 2007 infieren que, con un $\mathrm{R}^{2}$ de 0,99 y 0,97 , se podría producir un incremento de 0,0021 y $0,0043 \mathrm{cmol} / \mathrm{kg}$ respectivamente, en la estrata 0-20 $\mathrm{cm}$ al aplicar $1 \mathrm{~kg}$ de $\mathrm{MgO} / \mathrm{ha}$. En tanto, en 2006, con un $\mathrm{R}^{2}$ de 0,64 , se produciría un aumento de $0,0004 \mathrm{cmol} / \mathrm{kg}$ al aplicar $1 \mathrm{~kg}$ de $\mathrm{MgO} / \mathrm{ha}$.

Considerando los años 2005 y 2007, el valor promedio de incremento de disponibilidad del nutriente en el suelo sería de $0,0032 \mathrm{cmol} / \mathrm{kg}$, por cada kilo de magnesio aplicado. Con los resultados obtenidos se podría establecer la siguiente ecuación de cálculo de dosis de magnesio para un suelo andisol de la serie Freire:

Dosis de $\mathrm{MgO} / \mathrm{ha}=$ Valor deseado - Valor del suelo $/$ 0,0032

A modo de ejemplo, si el valor óptimo para un determinado cultivo en un suelo andisol fuera de 0,35 $\mathrm{cmol} / \mathrm{kg}$ y el suelo tuviera $0,25 \mathrm{cmol} / \mathrm{kg}$, habría que aplicar una dosis de 31 kilos de $\mathrm{MgO} /$ ha para alcanzar el valor deseado, al considerar la estrata $0-20 \mathrm{~cm}$.

Al respecto, cabe hacer presente que gran parte de los suelos andisoles de la región cerealera del país presentan niveles de magnesio que oscilan entre 0,4 a $0,8 \mathrm{cmol} / \mathrm{kg}$, y que en los suelos ultisoles este valor es mayor, superando por lo tanto el nivel de suficiencia señalado por Rodríguez et al. (2001).

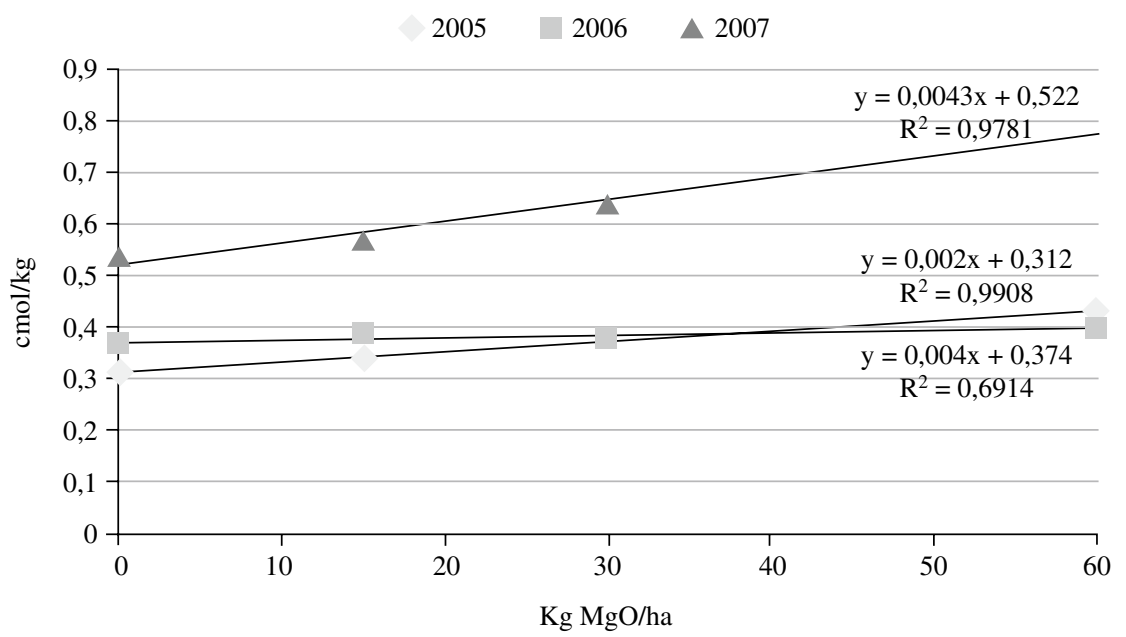

Figura 3. Efecto de las distintas dosis de magnesio evaluadas en la disponibilidad del nutriente en el suelo, en los años 2005, 2006 y 2007. 


\section{Conclusiones}

- No se produjeron diferencias significativas de rendimiento por efecto de las distintas dosis de magnesio en ninguno de los años evaluados, en un suelo andisol con niveles de magnesio de intercambio de 0,35 a $0,45 \mathrm{cmol} / \mathrm{kg}$. Con tal disponibilidad de $\mathrm{Mg}$ en el suelo no se produjeron, en general, incrementos en el contenido de gluten húmedo.

- De acuerdo a los resultados obtenidos en un suelo andisol de la serie Freire, se podría producir en promedio un incremento de 0,0032 $\mathrm{cmol} / \mathrm{kg}$ de magnesio en la estrata 0-20 al aplicar $1 \mathrm{~kg}$ de $\mathrm{MgO} / \mathrm{ha}$.

\section{Literatura Citada}

Dupont, F.M.; Altenbach, S.B.

2003 Molecular and biochemical impacts of environmenta factors on wheat grain development and protein synthesis. Journal of Cereal Science 38, 133-146.

Flaete, N.

2005 Combined nitrogen and sulphur fertilisation and its effect on wheat quality and protein composition measured by SE-FPLC and proteomics. Journal of Cereal Science 41 (2005) 357-369, Department of Plant and Environmental Sciences, Agricultural University of Norway, P.O. Box 5003, N-1432 A, Norway.

Hart, J.

2000 Winter Wheat. Fertilizer guide $N^{\circ}$ 9. Oregon State University (OSU). Available on www.eesc.orst.edu

Melgar, R.

2005 Trigo: Fertilización con potasio, azufre y magnesio. Documento del Proyecto "Fertilizar", INTA Pergamino, Argentina.

Mella, A. y Kühne, G.

1985 Sistema de descripción de las familias, asociaciones y series de suelos derivados de materiales piroclásticos de la zona central-sur de Chile. En: J. Tosso (Ed). Suelos Volcánicos de Chile. INIA. Ministerio de Agricultura. Santiago. Chile. Cap 1, pp. 25-95.

Rodríguez, J.

1993 La fertilización de los cultivos, un método racional. Colección en Agricultura, Pontificia Universidad Católica de Chile, Santiago, Chile. 291 p.

Rodríguez, J.; Pinochet, D. y Matus, F.

2001 La fertilización de los cultivos. Impreso en LOM Ediciones, Santiago, Chile. 117 p.

Ross, $\mathrm{M}$

2006 El magnesio en el cultivo del cereal. Disponible en www.kali-gmbh.com.
Rouanet, J.

1989 Clasificación agroclimática de la IX Región. Segunda aproximación. Investigación y progreso Agropecuario. Carillanca 2 (4): 25-27.

Singh, R. y Pathak, R.

2003 Response of wheat (Triticum aestivum) to integrated nutrition of $\mathrm{K}, \mathrm{Mg}, \mathrm{Zn}, \mathrm{S}$ and biofertilization. Journal of the Indian Society of Soil Science. 2003, vol. 51, nº 1, pp. 56-60.

SPSS

2004 Statistical Package for the Social Sciences (SPSS). SPSS Base 12.0 User's Guide for Windows. SPSS Inc., Chicago, Illinois, USA. Disponible en http://www.spss.com//spss

Tea, I.; Genter, T.; Violleau, F.; Kleiber, D.

2005 Changes in the glutathione thiol-disulfide status in wheat grain by foliar sulphur fertilization: consequences for the rheological properties of dough. Journal of Cereal Science 41, 305-315. Ecole Supérieure d'Agriculture de Purpan, Toulouse, France.

Tisdale, S. y Nelson, W.

1993 Soil fertility and fertilizers. MacMillan Publishing Company. New York.

Wieser, $\mathrm{H}$.

2004 Influence of sulphur fertilisation on quantities and proportions of gluten protein types in wheat flour. German Research Centre of Food Chemistry, Kürt-Hess-Institute, Lichtenbergstraße 4, D-85748 Garching, Germany. Journal of Cereal Science 40 (2004) 239-244.

Zadoks, J.; Chang, T. and Konzak, C.

1974 Código decimal para los estados de crecimiento de cereales. Weed Research. 14: 415-421.

Zhao, F.J.

1999 Sulphur Assimilation and Effects on Yield and Quality of Wheat. Journal of Cereal Science 30 (1999) 1-17. Soil Science Department and Biochemistry and Physiology Department, IACR-Rothamsted, Harpenden, Hertfordshire, AL5 2JQ, U.K. 